Bulletin de la Société Royale des Sciences de Liège, Vol. 85, 2016, p. 263 - 275

\title{
EFFET DE COMPOSÉS CALCIQUES INORGANIQUES SUR LE DÉVELOPPEMENT IN VITRO DE MOISISSURES ISOLÉES D'AGRUMES APRÈS LA RÉCOLTE
}

Manuscrit reçu le 25 août 2016 et accepté le 8 décembre 2016

\author{
Khaled ATTRASSI $^{(1)}$ et Mohamed RAHOUTI ${ }^{(2)}$
}

(1) Laboratoire Science de la vie et de la terre, CRMEF (Centre Régional des Métiers de l'Éducation et de la Formation), 245, Kénitra, Maroc. attrassi2@ yahoo.fr

(2) Université Mohammed V, Faculté des Sciences. Laboratoire de Botanique, Mycologie et Environnement. MA-10040 Rabat (Maroc).

\section{Résumé}

L'efficacité in vitro de silicate, d'hydroxyde, l'oxyde, du sulfate et d'hypochlorite de calcium a été testée sur le développement des champignons responsables de la pourriture des fruits d'agrumes en post-récolte.

Les composés calciques inorganiques ont été testés in vitro à 200, 400, 600, 800 et 1000 ppm contre Alternaria alternata, Aspergillus niger, Fusarium oxysporum, Penicillium digitatum, Rhizopus stolonifer, Penicillium italicum isolées à partir de deux variétés d'agrumes Clémentines et Oranges. Le silicate, l'hydroxyde et l'oxyde de calcium se sont avérés efficaces sur les isolats de $F$. oxysporum, d'A. alternata, d'A. niger, de $P$. digitatum, de $R$. stolonifer et de $P$. italicum à $1000 \mathrm{ppm}$ avec des pourcentages d'inhibition compris entre $66,7 \%$ et $85,7 \%$ pour la croissance mycélienne, 62,8 et $92,4 \%$ pour la production des conidies et 82,8 et $98,8 \%$ pour leur germination. Les champignons testés sont moyennement sensibles au sulfate de calcium alors que l'hypochlorite de calcium est sans action.

Le silicate, l'hydroxyde et l'oxyde de calcium sont les composés calciques inorganiques les plus efficaces in vitro contre les champignons responsables des pourritures des fruits d'agrumes en post-récolte. Les résultats obtenus vont permettre de s'orienter vers la lutte par les composés calciques inorganiques contre les pathogènes responsables des pourritures des fruits d'agrumes en post-récolte, avant l'entreposage, en raison de son efficacité contre les maladies d'origine fongiques.

Mots clés: Pourriture, champignon, fruits d'agrume, post récolte, sels de calcium.

\section{Abstract}

The in vitro efficacy of silicate, hydroxide, oxide, sulfate and calcium hypochlorite were tested on the development of fungi responsible for storage rot in citrus fruit.

Inorganic calcic compounds were tested in vitro at 200, 400, 600, 800 and 1000 ppm against Alternaria alternata, Aspergillus niger, Fusarium oxysporum, Penicillium digitatum, Rhizopus stolonifer, Penicillium italicum isolated from two varieties of citrus Clémentines and Oranges. In vitro, silicate, hydroxide and calcium oxide were effective at $1000 \mathrm{ppm}$ on the $F$. oxysporum, d'A. alternata, d'A. niger, de $P$. digitatum, de $R$. stolonifer et de $P$. italicum isolates. The inhibition percentage was $66.7 \%-85.7 \%$ for mycelial growth, $62.8-92.4 \%$ for conidia production and $82.8-98.8 \%$ for conidia germination. Calcium sulfate was moderately 
effective against the three stages of the fungi life cycle whereas calcium hypochlorite was ineffective.

Silicate, hydroxide and oxide are the inorganic calcic compounds most effective against the fungal species responsible for storage rot in citrus fruit. The results obtained point to research on inorganic calcic compounds control against the pathogens responsible for post-harvest rots in citrus fruit, before storage, because of their effectiveness against fungal diseases.

Keywords: Citrus fruit, rot, fungi, storage, calcium salts.

\section{INTRODUCTION}

L'agrumiculture demeure le secteur agricole exportateur le plus important au Maroc. Elle joue un rôle primordial dans le développement économique national (Anonyme, 2003).Toutefois, les agrumes sont exposés à de nombreux problèmes phytosanitaires, dont la pourriture verte causée par Penicillium digitatum Sacc. qui est responsable des pertes économiquement significatives au Maroc (Elkhamass et al., 1994) et dans le monde (Bancroft et al., 1984 ; Eckert \& Eaks, 1989). La lutte contre la pourriture verte est principalement basée sur l'utilisation de traitements fongicides, dont les plus fréquents sont le thiabendazole (TBZ) et l'imazalil (IMZ). Ces traitements chimiques présentent de nombreux inconvénients, dont la persistance des résidus sur les fruits traités et l'apparition de souches résistantes aux produits fongicides utilisés (El-Goorani et al., 1984 ; Eckert, 1990 ; Bus et al., 1997 ; Eckert et al., 1994), ce qui nécessite l'augmentation des doses ou la recherche de nouvelles molécules actives (Miller et al., 1988), en plus de l'impact néfaste des résidus chimiques sur la santé du consommateur et sur l'environnement (Chalutz \& Wilson, 1990). L'apparition de souches de $P$. digitatum résistantes à ces fongicides et la nécessité de préserver la santé du consommateur et le respect de l'environnement imposent la recherche d'autres moyens pour contrôler les maladies des fruits en post-récolte. Malheureusement, l'usage fréquent des fongicides a été responsable de l'apparition de la résistance des champignons pathogènes et la prise de conscience du consommateur vis-à-vis de la contamination des denrées alimentaires par les résidus de fongicides à orienter la recherche vers d'autre moyens de lutte. Les produits alternatifs qui sont sûrs, efficaces et économiques sont nécessaires pour contrôler les maladies de post de récolte. Des additifs alimentaires ont été signalés pour le contrôle de la pourriture d'entreposage des récoltes de fruits et légumes (Punja et Gaye 1993). Certains composés prometteurs sont les sels de bicarbonate (Aharoni et al., 1997 ; Smilanick et al., 1999), les acétates et les sels de molybdate (Punja et Gaye 1993), l'iode (Bokshi, 2008), le silicate de sodium (Bi et al. 2006) et les isothiocyanates (Troncoso-Rojas et al., 2009). Zemmouri et al. (2011) ont montré l'effet in vitro et in vivo du propionate, du chlorure et du nitrate de calcium sur Fusarium oxysporum et Alternaria alternata. Le propionate de calcium s'est avéré efficace sur les trois stades du cycle de vie des deux champignons et sur le développement des pourritures sur les fruits de melon Cantaloup et Galia. Plusieurs études ont utilisé différents composés de sels pour contrôler les maladies de post-récolte d'agrumes (Arslan et al., 2006; Nigro et al., 2006; Arslan et al., 2009; Khamis et al., 2012 ). De plus, Askame et al., (20011) ont montré les effets des sels organiques sur le développement de Penicillium italicum, agent causal de la pourriture d'agrumes. D'un autre côté, Martin-Diana et al. (2007) ont trempé les 
fruits dans des solutions de calcium, peut améliorer leur qualité nutritionnelle durant la conservation et accroître la durée de leur vie en maintenant leur fermeté. L'objectif de ce travail est de tester l'efficacité de cinq autres sels de calcium in vitro sur les trois stades du cycle de vie de Alternaria alternata, Aspergillus niger, Fusarium oxysporum, Penicillium digitatum, Rhizopus stolonifer, Penicillium italicum et de voir l'effet de ceux qui se sont montré les plus efficaces sur le développement des pourritures provoquées par ces six champignons.

\section{MATÉRIEL ET MÉTHODES}

\section{Matériel fongique:}

Les espèces fongiques Alternaria alternata, Aspergillus niger, Fusarium oxysporum, Penicillium digitatum, Rhizopus stolonifer, Penicillium italicum ont été isolés respectivement à partir des lésions des fruits de deux variétés d'agrumes; clémentines d'un clémentinier (Citrus clementina) oranges foncées, petites et de texture onctueuse et oranges et d'un oranger (Citrus sinensis), de couleur orange, de taille moyenne et réniforme de chambres froides de Kénitra avec six mois de temps de stockage. Ils ont été maintenus sur le milieu de culture PSA (Potato Sucrose Agar : $200 \mathrm{~g}$ pomme de terre, $15 \mathrm{~g}$ Agar-agar, $20 \mathrm{~g}$ saccharose, $1000 \mathrm{ml}$ d'eau distillée) à $25^{\circ} \mathrm{C}$ et à l'obscurité.

Composés calciques inorganiques testés: Cinq sels de calcium, le silicate de calcium $\mathrm{Ca}_{2} \mathrm{SiO}_{4}$, l'hydroxyde de calcium $\mathrm{Ca}(\mathrm{OH})_{2}$, l'oxyde de calcium $\mathrm{CaO}$, le sulfate de calcium $\mathrm{CaSO}_{4}, 2 \mathrm{H}_{2} \mathrm{O}$ et l'hypochlorite de calcium $\mathrm{Ca}(\mathrm{ClO})_{2}$ ont été testés à des concentrations croissantes de 200 , 400, 600, 800 et 1000 ppm.

\section{Action sur les trois stades de vie du cycle:}

Les sels de calcium aux différentes concentrations ont été incorporés au milieu de culture PSA maintenu en surfusion à $50^{\circ} \mathrm{C}$. Un disque mycélien de $5 \mathrm{~mm}$ de diamètre prélevé sur une culture jeune de champignon a été déposé au centre de chacune des boîtes de Pétri de $90 \mathrm{~mm}$ de diamètre contenant $15 \mathrm{ml}$ de milieu de culture seul pour le témoin ou additionné des sels de calcium à différentes concentrations. Après dix jours d'incubation à l'obscurité et à $25^{\circ} \mathrm{C}$, la croissance mycélienne a été estimée en mesurant la moyenne de deux diamètres perpendiculaires de chaque culture. La production des conidies est estimée sur les boîtes de Pétri ayant servi à mesurer la croissance mycélienne. Pour chaque traitement, quatre rondelles de $5 \mathrm{~mm}$ de diamètre ont été prélevées, placées dans un tube à essai stérile contenant $1 \mathrm{ml}$ d'eau distillée stérile et agitées au vortex pendant quelques secondes. Le nombre moyen de conidies a été estimé à l'aide d'une cellule de Malassez. La germination des conidies est estimée en étalant $0,1 \mathrm{ml}$ de la suspension conidiale à 103 conidies/ml obtenue à partir des différents champignons à la surface des boîtes de Pétri de $90 \mathrm{~mm}$ de diamètre contenant $15 \mathrm{ml}$ d'eau gélosée stérile seule pour le témoin ou additionnée des différents composés calciques inorganiques à différentes concentrations. Après 24 heures d'incubation à l'obscurité et à $25^{\circ} \mathrm{C}$, le comptage des conidies germées a été fait sur toute la surface de chaque boîtes de Pétri. Trois répétitions ont été réalisées pour chacun des traitements. Le pourcentage 
d'inhibition des trois stades du cycle de vie des champignons est calculé par rapport au témoin selon la formule suivante:

$$
\mathrm{PI}=\frac{\mathrm{A}-\mathrm{B}}{\mathrm{A}} \times 100
$$

PI : Pourcentage d'inhibition

A: Croissance mycélienne, production des conidies ou la germination des champignons testés sur le milieu de culture sans sel de calcium.

B: Croissance mycélienne, production des conidies ou la germination des champignons testés sur le milieu de culture additionné de composés calciques inorganiques.

\section{RÉSULTATS}

Les différents composés calciques inorganiques ont montré in vitro une efficacité variable sur les trois stades du cycle de vie des espèces fongiques responsables de la pourriture de post récolte des agrumes. Les pourcentages d'inhibition de la croissance mycélienne les plus importants sont obtenus avec l'hydroxyde, le silicate et l'oxyde de calcium à 1000 ppm (Tableau 1). Ils atteignent $85,7 \%$ pour l'isolat de Fusarium oxysporum, $85 \%$ pour l'isolat de Penicillium italicum et $83,6 \%$ pour Trichothecium roseum. En présence de silicate et l'hydroxyde de calcium, l'inhibition dépasse $40 \%$ dès $400 \mathrm{ppm}$ pour tous les isolats des champignons testés.

En présence du sulfate de calcium, la croissance mycélienne des champignons testés est moyennement efficace, les pourcentages d'inhibition obtenus sont compris entre $35,5 \%$ et 48,2\% à 1000 ppm. L'hypochlorite de calcium s'est montré inefficace dans l'inhibition de la croissance mycélienne de tous les champignons testés. Les $\mathrm{CI}_{50}$ ne dépassent pas 409 ppm pour l'hydroxyde de calcium, $422 \mathrm{ppm}$ pour le silicate de calcium et $616 \mathrm{ppm}$ pour l'oxyde de calcium (Tableau 2). Le sulfate et l'hypochlorite de calcium sont inefficaces sur le développement mycélien de tous les champignons testés. Le silicate, l'hydroxyde et l'oxyde de calcium présentent une efficacité importante. Les pourcentages d'inhibition atteignent 92,4 $\%$ pour Fusarium oxysporum, 89,8 \% pour Alternaria alternata et 88,7\% pour Trichothecium roseum à $1000 \mathrm{ppm}$

(Tableau 3). Les champignons testés sont très résistants au sulfate et à l'hypochlorite de calcium. 
Tableau 1: Comparaison des moyennes des pourcentages d'inhibition de la croissance mycélienne des isolats des moisissures d'agrumes en présence de cinq composés calciques inorganiques à différente concentration

\begin{tabular}{|c|c|c|c|c|c|c|c|}
\hline \multicolumn{2}{|c|}{$\begin{array}{c}\text { Sels de calcium } \\
(\mathbf{p p m})\end{array}$} & $\begin{array}{c}F . \\
\text { oxysporum }\end{array}$ & $\begin{array}{c}\text { A. } \\
\text { alternata }\end{array}$ & $\begin{array}{c}\text { A. } \\
\text { niger }\end{array}$ & $\begin{array}{c}P . \\
\text { digitatum }\end{array}$ & $\begin{array}{c}P . \\
\text { italicum }\end{array}$ & $\begin{array}{c}R . \\
\text { stolonifer }\end{array}$ \\
\hline \multirow[t]{5}{*}{$\mathrm{Ca}_{2} \mathrm{SiO}_{4}$} & 200 & $41,2 \mathrm{e}$ & $30,5 \mathrm{e}$ & $34,8 \mathrm{e}$ & $29,7 \mathrm{e}$ & $33,3 \mathrm{e}$ & $36,7 \mathrm{e}$ \\
\hline & 400 & $55,8 \mathrm{~d}$ & $44,1 d$ & $51,5 \mathrm{~d}$ & $46,0 \mathrm{~d}$ & $53,3 d$ & $50,7 d$ \\
\hline & 600 & $66,6 c$ & $55,2 \mathrm{c}$ & $61,3 c$ & $57,4 c$ & $66,4 \mathrm{c}$ & $62,6 \mathrm{c}$ \\
\hline & 800 & $75,4 b$ & $71,3 b$ & $68,4 b$ & $73,3 b$ & $74,9 \mathrm{~b}$ & $71,3 b$ \\
\hline & 1000 & $85,8 \mathrm{a}$ & $77,7 a$ & $80,8 \mathrm{a}$ & $81,4 a$ & $85,5 a$ & $84,1 \mathrm{a}$ \\
\hline \multirow[t]{5}{*}{$\mathrm{Ca}(\mathrm{OH})_{2}$} & 200 & $31,7 \mathrm{e}$ & $33,5 \mathrm{e}$ & $33,6 \mathrm{e}$ & $35,5 \mathrm{e}$ & $33,2 \mathrm{e}$ & $30,4 \mathrm{e}$ \\
\hline & 400 & $49,2 d$ & $47,1 d$ & $45,3 d$ & $47,6 \mathrm{~d}$ & $46,1 d$ & $44,8 \mathrm{~d}$ \\
\hline & 600 & $61,8 \mathrm{c}$ & $58,4 \mathrm{c}$ & $60,1 c$ & $61,6 c$ & $60,1 c$ & $59,7 \mathrm{c}$ \\
\hline & 800 & $70,5 b$ & $71,6 b$ & $72,0 \mathrm{~b}$ & $73,4 b$ & $71,5 b$ & $72,3 b$ \\
\hline & 1000 & $78,5 a$ & $80,4 a$ & $81,5 a$ & $81,3 a$ & $80,8 \mathrm{a}$ & $82,4 a$ \\
\hline \multirow[t]{5}{*}{$\mathrm{CaO}$} & 200 & $24,3 \mathrm{e}$ & $27,7 \mathrm{e}$ & $30,4 \mathrm{e}$ & $33,8 \mathrm{e}$ & $32,6 \mathrm{e}$ & $22,1 \mathrm{e}$ \\
\hline & 400 & $37,5 \mathrm{~d}$ & $41,8 \mathrm{~d}$ & $41,4 d$ & $45,1 d$ & $46,0 \mathrm{~d}$ & $30,8 \mathrm{~d}$ \\
\hline & 600 & $49,2 \mathrm{c}$ & $51,8 \mathrm{c}$ & $49,7 c$ & $53,5 \mathrm{c}$ & $53,8 \mathrm{c}$ & $44,8 \mathrm{c}$ \\
\hline & 800 & $60,2 b$ & $58,4 b$ & $64,0 \mathrm{~b}$ & $63,5 b$ & $61,8 b$ & $57,5 b$ \\
\hline & 1000 & $70,0 a$ & $69,1 \mathrm{a}$ & $68,9 a$ & $73,4 a$ & $70,5 a$ & $71,5 \mathrm{a}$ \\
\hline \multirow[t]{5}{*}{$\mathrm{CaSO}_{4}, 2 \mathrm{H} 2 \mathrm{O}$} & 200 & $12,9 \mathrm{e}$ & $8,0 \mathrm{e}$ & $6,5 \mathrm{e}$ & $10,7 \mathrm{e}$ & $8,8 \mathrm{e}$ & $7,0 \mathrm{e}$ \\
\hline & 400 & $18,8 \mathrm{~d}$ & $17,1 d$ & $14,0 \mathrm{~d}$ & $16,2 d$ & $14,7 d$ & $13,8 \mathrm{~d}$ \\
\hline & 600 & $28,6 \mathrm{c}$ & $24,7 \mathrm{c}$ & $21,8 \mathrm{c}$ & $23,9 c$ & $25,9 c$ & $20,6 c$ \\
\hline & 800 & $36,5 b$ & $31,3 b$ & $29,4 b$ & $30,7 b$ & $34,7 b$ & $29,4 b$ \\
\hline & 1000 & $47,9 a$ & $40,6 a$ & $37,6 a$ & $38,4 a$ & $43,4 a$ & $35,5 a$ \\
\hline \multirow[t]{5}{*}{$\mathrm{Ca}(\mathrm{ClO})_{2}$} & 200 & $4,6 \mathrm{~d}$ & $5,5 \mathrm{dd}$ & $3,6 \mathrm{e}$ & $4,0 \mathrm{e}$ & $6,2 \mathrm{c}$ & $3,2 d$ \\
\hline & 400 & $4,8 \mathrm{~d}$ & $5,3 d$ & $5,7 \mathrm{de}$ & $6,5 d$ & $4,8 \mathrm{~d}$ & $5,8 \mathrm{c}$ \\
\hline & 600 & $7,0 \mathrm{c}$ & $6,6 \mathrm{c}$ & $5,4 \mathrm{~cd}$ & $8,8 \mathrm{c}$ & $6,7 \mathrm{c}$ & $9,3 \mathrm{~b}$ \\
\hline & 800 & $9,6 b$ & $9,6 b$ & $9,0 \mathrm{~b}$ & $10,5 b$ & $9,4 b$ & $10,2 b$ \\
\hline & 1000 & $14,3 \mathrm{a}$ & $12,4 a$ & $10,5 a$ & $13,6 a$ & $15,3 a$ & $16,4 a$ \\
\hline
\end{tabular}

Pour chaque isolat et chaque sel, deux résultats lus sur une même colonne diffèrent significativement au seuil de 5\% s'ils ne sont affectés d'aucune lettre en commun.

A. alternata: Alternaria alternata, A. niger: Aspergillus niger, F. oxysporum: Fusarium oxysporum, $\boldsymbol{P}$. digitatum: Penicillium digitatum, $\boldsymbol{R}$. stolonifer: Rhizopus stolonifer, $\boldsymbol{P}$.

italicum: Penicillium italicum 
Tableau 2 : $\mathrm{CI}_{50}$ et $\mathrm{CI}_{90}(\mathrm{ppm})$ de la croissance mycélienne des champignons testés en présence de composés calciques inorganiques.

\begin{tabular}{|c|c|c|c|c|c|c|c|c|c|c|c|c|}
\hline \multirow{2}{*}{$\begin{array}{c}\text { Sels de } \\
\text { calcium }\end{array}$} & \multicolumn{10}{|c|}{ Champignons } \\
\cline { 2 - 15 } & \multicolumn{2}{|c|}{ Aa } & \multicolumn{2}{c|}{ An } & \multicolumn{2}{c|}{ Pd } & \multicolumn{2}{|c|}{ Pi } & \multicolumn{3}{|c|}{ Fo } & \multicolumn{2}{c|}{ Rs } \\
\cline { 2 - 15 } & $\mathbf{C I}_{\mathbf{5 0}}$ & $\mathbf{C I}_{\mathbf{9 0}}$ & $\mathbf{C I}_{\mathbf{5 0}}$ & $\mathbf{C I}_{\mathbf{9 0}}$ & $\mathbf{C I}_{\mathbf{5 0}}$ & $\mathbf{C I}_{\mathbf{9 0}}$ & $\mathbf{C I}_{\mathbf{5 0}}$ & $\mathbf{C I}_{\mathbf{9 0}}$ & $\mathbf{C I}_{\mathbf{5 0}}$ & $\mathbf{C I}_{\mathbf{9 0}}$ & $\mathbf{C I}_{\mathbf{5 0}}$ & $\mathbf{C I}_{\mathbf{9 0}}$ \\
\hline Hydroxyde & 408 & $>1000$ & 389 & $>1000$ & 366 & $>1000$ & 394 & $>1000$ & 398 & $>1000$ & 398 & $>1000$ \\
\hline Sulfate & $>1000$ & $>1000$ & $>1000$ & $>1000$ & $>1000$ & $>1000$ & $>1000$ & $>1000$ & $>1000$ & $>1000$ & $>1000$ & $>1000$ \\
\hline Silicate & 404 & $>1000$ & 357 & $>1000$ & 424 & $>1000$ & 345 & $>1000$ & 296 & $>1000$ & 347 & $>1000$ \\
\hline Oxyde & 470 & $>1000$ & 516 & $>1000$ & 445 & $>1000$ & 478 & $>1000$ & 554 & $>1000$ & 618 & $>1000$ \\
\hline Hypochlorite & $>1000$ & $>1000$ & $>1000$ & $>1000$ & $>1000$ & $>1000$ & $>1000$ & $>1000$ & $>1000$ & $>1000$ & $>1000$ & $>1000$ \\
\hline
\end{tabular}

Aa : Alternaria alternata, An :Aspergillus niger, Fo :Fusarium oxysporum, Pd:Penicillium digitatum, Rs:Rhizopus stolonifer, Pi :Penicillium italicum

Pour tous les champignons testés, les $\mathrm{CI}_{50}$ varient entre 235 et 363 pour le silicate de calcium, 285 et $313 \mathrm{ppm}$ pour l'hydroxyde de calcium et entre 303 et $721 \mathrm{ppm}$ pour l'oxyde de calcium (Tableau 4). Le sulfate et l'hypochlorite de calcium n'ont pas d'effet inhibiteur avec des $\mathrm{CI}_{50}$ supérieures aux concentrations testées. Les pourcentages d'inhibition de la germination des conidies les plus importantes sont obtenues avec le silicate, l'hydroxyde et l'oxyde de calcium, elles varient entre 82,8 et 98,8 \% à 1000 ppm (Tableau 5). En présence de l'hydroxyde de calcium, l'inhibition dépasse $40 \%$ dès 200 ppm pour tous les isolats des champignons testés. La germination des conidies des champignons est sensiblement affectée en présence du sulfate de calcium (les pourcentages d'inhibition obtenus sont compris entre 33,3 et $52,3 \%$ ) et l'hypochlorite de calcium n'est pas efficaces. Les $\mathrm{CI}_{50}$ de silicate, l'hydroxyde et l'oxyde de calcium varient respectivement entre 228 et 331 ppm, 267 et 346 ppm et entre 271 et 446 ppm (Tableau 6). Les $\mathrm{CI}_{50}$ des autres produits à savoir l'hypochlorite et le sulfate de calcium sont supérieures aux concentrations testées. 
Tableau 3 : Comparaison des moyennes des pourcentages d'inhibition de la production des conidies des isolats des moisissures d'agrumes en présence de cinq composés calciques inorganiques à différente concentration

\begin{tabular}{|c|c|c|c|c|c|c|c|}
\hline \multicolumn{2}{|c|}{$\begin{array}{c}\text { Sels de calcium } \\
\text { (ppm) }\end{array}$} & $\begin{array}{c}F . \\
\text { oxysporum }\end{array}$ & $\begin{array}{c}\text { A. } \\
\text { alternata }\end{array}$ & $\begin{array}{c}\text { A. } \\
\text { niger }\end{array}$ & $\begin{array}{c}P . \\
\text { digitatum }\end{array}$ & $\begin{array}{c}P . \\
\text { italicum }\end{array}$ & $\begin{array}{c}R . \\
\text { stolonifer }\end{array}$ \\
\hline \multirow{5}{*}{$\mathrm{Ca}_{2} \mathrm{SiO}_{4}$} & 200 & $44,2 \mathrm{e}$ & $35,5 \mathrm{e}$ & $38,8 \mathrm{e}$ & $34,7 \mathrm{e}$ & $42,3 \mathrm{e}$ & $39,7 \mathrm{e}$ \\
\hline & 400 & $63,8 \mathrm{~d}$ & $49,1 d$ & $53,5 d$ & $48,0 \mathrm{~d}$ & $56,3 d$ & $52,7 d$ \\
\hline & 600 & $72,6 c$ & $62,2 \mathrm{c}$ & $64,3 c$ & $60,4 c$ & $68,4 c$ & $65,6 c$ \\
\hline & 800 & $84,4 b$ & $75,3 b$ & $78,4 b$ & $75,3 b$ & $80,9 b$ & $73,3 b$ \\
\hline & 1000 & $92,8 \mathrm{a}$ & $82,7 a$ & $87,8 \mathrm{a}$ & $85,4 a$ & $88,5 a$ & $85,1 \mathrm{a}$ \\
\hline \multirow[t]{5}{*}{$\mathrm{Ca}(\mathrm{OH})_{2}$} & 200 & $38,7 \mathrm{e}$ & $41,5 \mathrm{e}$ & $40,6 \mathrm{e}$ & $40,5 \mathrm{e}$ & $43,2 \mathrm{e}$ & $39,4 \mathrm{e}$ \\
\hline & 400 & $53,2 d$ & $57,1 d$ & $55,3 d$ & $57,6 \mathrm{~d}$ & $56,1 d$ & $54,8 \mathrm{~d}$ \\
\hline & 600 & $68,8 \mathrm{c}$ & $64,4 \mathrm{c}$ & $65,1 \mathrm{c}$ & $67,6 c$ & $66,1 \mathrm{c}$ & $65,7 \mathrm{c}$ \\
\hline & 800 & $84,5 b$ & $78,6 b$ & $79,0 \mathrm{~b}$ & $80,4 b$ & $78,5 b$ & $87,3 b$ \\
\hline & 1000 & $91,5 \mathrm{a}$ & $84,4 a$ & $86,5 \mathrm{a}$ & $88,3 \mathrm{a}$ & $89,8 \mathrm{a}$ & $82,4 a$ \\
\hline \multirow[t]{5}{*}{$\mathrm{CaO}$} & 200 & $18,3 \mathrm{e}$ & $40,7 \mathrm{e}$ & $37,4 \mathrm{e}$ & $41,8 \mathrm{e}$ & $43,6 \mathrm{e}$ & $17,1 \mathrm{e}$ \\
\hline & 400 & $34,5 d$ & $51,8 d$ & $49,4 d$ & $54,1 d$ & $52,0 \mathrm{~d}$ & $30,8 \mathrm{~d}$ \\
\hline & 600 & $48,2 \mathrm{c}$ & $64,8 \mathrm{c}$ & $60,7 \mathrm{c}$ & $63,5 c$ & $63,8 \mathrm{c}$ & $38,8 \mathrm{c}$ \\
\hline & 800 & $53,2 b$ & $75,4 \mathrm{~b}$ & $72,0 b$ & $70,5 b$ & $71,8 b$ & $54,5 b$ \\
\hline & 1000 & $68,0 \mathrm{a}$ & $63,1 \mathrm{a}$ & $86,9 a$ & $79,4 a$ & $80,5 a$ & $66,5 a$ \\
\hline \multirow[t]{5}{*}{$\mathrm{CaSO}_{4}, 2 \mathrm{H}_{2} \mathrm{O}$} & 200 & $10,9 \mathrm{e}$ & $6,0 \mathrm{e}$ & $7,5 \mathrm{e}$ & $7,7 \mathrm{e}$ & $9,8 \mathrm{e}$ & $7,0 \mathrm{e}$ \\
\hline & 400 & $14,8 \mathrm{~d}$ & $14,1 d$ & $12,0 \mathrm{~d}$ & $16,2 d$ & $18,7 d$ & $13,8 \mathrm{~d}$ \\
\hline & 600 & $24,6 \mathrm{c}$ & $20,7 \mathrm{c}$ & $21,8 \mathrm{c}$ & $19,9 \mathrm{c}$ & $27,9 \mathrm{c}$ & $20,6 c$ \\
\hline & 800 & $33,5 b$ & $28,3 b$ & $27,4 b$ & $30,7 b$ & $37,7 b$ & $26,4 b$ \\
\hline & 1000 & $45,9 \mathrm{a}$ & $34,6 \mathrm{a}$ & $32,6 \mathrm{a}$ & $31,4 \mathrm{a}$ & $39,4 a$ & $37,5 \mathrm{a}$ \\
\hline \multirow[t]{5}{*}{$\mathrm{Ca}(\mathrm{ClO})_{2}$} & 200 & $6,6 \mathrm{~d}$ & $5,5 \mathrm{dd}$ & $3,6 \mathrm{e}$ & $4,0 \mathrm{e}$ & $5,2 \mathrm{c}$ & $3,7 d$ \\
\hline & 400 & $5,8 \mathrm{~d}$ & $4,3 \mathrm{~d}$ & $5,7 \mathrm{de}$ & $6,5 \mathrm{~d}$ & $6,8 \mathrm{~d}$ & $6,8 \mathrm{c}$ \\
\hline & 600 & $9,0 \mathrm{c}$ & $5,6 \mathrm{c}$ & $7,4 \mathrm{~cd}$ & $5,8 \mathrm{c}$ & $6,7 \mathrm{c}$ & $9,3 b$ \\
\hline & 800 & $11,6 b$ & $6,6 b$ & $9,0 \mathrm{~b}$ & $9,5 b$ & $6,4 b$ & $10,2 b$ \\
\hline & 1000 & $15,3 \mathrm{a}$ & $10,4 a$ & $10,5 \mathrm{a}$ & $12,6 \mathrm{a}$ & $9,3 \mathrm{a}$ & $12,4 \mathrm{a}$ \\
\hline
\end{tabular}

Pour chaque isolat et chaque sel, deux résultats lus sur une même colonne diffèrent significativement au seuil de 5\% s'ils ne sont affectés d'aucune lettre en commun.

A. alternata: Alternaria alternata, A. niger: Aspergillus niger, F. oxysporum: Fusarium oxysporum, P. digitatum: Penicillium digitatum, $\boldsymbol{R}$. stolonifer: Rhizopus stolonifer, $\boldsymbol{P}$.

italicum: Penicillium italicum 
Bulletin de la Société Royale des Sciences de Liège, Vol. 85, 2016, p. 263 - 275

Tableau $4: \mathrm{CI}_{50}$ et $\mathrm{CI}_{90}(\mathrm{ppm})$ de la production des conidies des champignons testés en présence de composés calciques inorganiques.

\begin{tabular}{|c|c|c|c|c|c|c|c|c|c|c|c|c|}
\hline \multirow{3}{*}{$\begin{array}{l}\text { Sels de } \\
\text { calcium }\end{array}$} & \multicolumn{12}{|c|}{ Champignons } \\
\hline & \multicolumn{2}{|c|}{ Aa } & \multicolumn{2}{|c|}{ An } & \multicolumn{2}{|c|}{$\mathbf{P d}$} & \multicolumn{2}{|c|}{$\mathbf{P i}$} & \multicolumn{2}{|c|}{ Fo } & \multicolumn{2}{|c|}{ Rs } \\
\hline & $\mathrm{CI}_{50}$ & $\mathbf{C I}_{90}$ & $\mathrm{CI}_{50}$ & $\mathrm{CI}_{90}$ & $\mathrm{CI}_{50}$ & $\mathrm{CI}_{90}$ & $\mathrm{CI}_{50}$ & $\mathrm{CI}_{90}$ & $\mathrm{CI}_{50}$ & $\mathrm{CI}_{90}$ & $\mathrm{CI}_{50}$ & $\mathrm{CI}_{90}$ \\
\hline Hydroxyde & 314 & $>1000$ & 302 & $>1000$ & 286 & $>1000$ & 311 & $>1000$ & 308 & $>1000$ & 310 & $>1000$ \\
\hline Sulfate & $>1000$ & $>1000$ & $>1000$ & $>1000$ & $>1000$ & $>1000$ & $>1000$ & $>1000$ & $>1000$ & $>1000$ & $>1000$ & $>1000$ \\
\hline Silicate & 350 & $>1000$ & 317 & $>1000$ & 363 & $>1000$ & 285 & $>1000$ & 236 & $>1000$ & 326 & $>1000$ \\
\hline Oxyde & 316 & $>1000$ & 341 & $>1000$ & 311 & $>1000$ & 303 & $>1000$ & 634 & $>1000$ & 721 & $>1000$ \\
\hline Hypochlorite & $>1000$ & $>1000$ & $>1000$ & $>1000$ & $>1000$ & $>1000$ & $>1000$ & $>1000$ & $>1000$ & $>1000$ & $>1000$ & $>1000$ \\
\hline
\end{tabular}

Aa : Alternaria alternata, An :Aspergillus niger, Fo:Fusarium oxysporum, Pd:Penicillium digitatum, Rs:Rhizopus stolonifer, Pi :Penicillium italicum 
Tableau 5 : Comparaison des moyennes des pourcentages d'inhibition de la germination des conidies des isolats de trois moisissures d'agrume en présence de cinq composés calciques inorganiques à différentes concentrations.

\begin{tabular}{|c|c|c|c|c|c|c|c|}
\hline \multicolumn{2}{|c|}{$\begin{array}{c}\text { Sels de calcium } \\
\text { (ppm) }\end{array}$} & $\begin{array}{c}F . \\
\text { oxysporum }\end{array}$ & $\begin{array}{c}\text { A. } \\
\text { alternata }\end{array}$ & $\begin{array}{c}\text { A. } \\
\text { niger }\end{array}$ & $\begin{array}{c}P . \\
\text { digitatum }\end{array}$ & $\begin{array}{c}P . \\
\text { italicum }\end{array}$ & $\begin{array}{c}\text { R. } \\
\text { stolonifer }\end{array}$ \\
\hline \multirow[t]{5}{*}{$\mathrm{Ca}_{2} \mathrm{SiO}_{4}$} & 200 & $50,2 \mathrm{e}$ & $39,5 \mathrm{e}$ & $41,8 \mathrm{e}$ & $38,7 \mathrm{e}$ & $45,3 \mathrm{e}$ & $41,7 \mathrm{e}$ \\
\hline & 400 & $65,8 d$ & $53,1 d$ & $58,5 d$ & $52,0 \mathrm{~d}$ & $60,3 d$ & $57,7 d$ \\
\hline & 600 & $77,6 \mathrm{c}$ & $67,2 c$ & $71,3 \mathrm{c}$ & $64,4 c$ & $70,4 c$ & $69,6 c$ \\
\hline & 800 & $86,4 b$ & $76,3 b$ & $80,4 b$ & $79,3 b$ & $82,9 b$ & $80,3 b$ \\
\hline & 1000 & $92,8 \mathrm{a}$ & $85,7 a$ & $89,8 \mathrm{a}$ & $86,4 a$ & $92,5 \mathrm{a}$ & $92,1 \mathrm{a}$ \\
\hline \multirow[t]{5}{*}{$\mathrm{Ca}(\mathrm{OH})_{2}$} & 200 & $44,7 \mathrm{e}$ & $43,5 \mathrm{e}$ & $42,6 \mathrm{e}$ & $45,5 \mathrm{e}$ & $40,2 \mathrm{e}$ & $43,4 \mathrm{e}$ \\
\hline & 400 & $56,2 d$ & $57,1 d$ & $57,3 \mathrm{~d}$ & $58,6 \mathrm{~d}$ & $56,1 \mathrm{~d}$ & $54,8 d$ \\
\hline & 600 & $72,8 \mathrm{c}$ & $69,4 c$ & $70,1 \mathrm{c}$ & $70,6 c$ & $72,1 \mathrm{c}$ & $69,7 c$ \\
\hline & 800 & $84,5 b$ & $81,6 b$ & $83,0 \mathrm{~b}$ & $83,4 b$ & $84,5 b$ & $81,3 b$ \\
\hline & 1000 & $98,5 a$ & $90,4 a$ & $90,5 a$ & $93,3 a$ & $95,8 \mathrm{a}$ & $92,4 a$ \\
\hline \multirow[t]{5}{*}{$\mathrm{CaO}$} & 200 & $35,3 \mathrm{e}$ & $41,7 \mathrm{e}$ & $44,4 \mathrm{e}$ & $42,8 \mathrm{e}$ & $45,6 \mathrm{e}$ & $25,1 \mathrm{e}$ \\
\hline & 400 & $54,5 \mathrm{~d}$ & $56,8 \mathrm{~d}$ & $59,4 d$ & $60,1 d$ & $58,0 \mathrm{~d}$ & $40,8 \mathrm{~d}$ \\
\hline & 600 & $67,2 \mathrm{c}$ & $64,8 \mathrm{c}$ & $68,7 \mathrm{c}$ & $68,5 c$ & $66,8 c$ & $55,8 \mathrm{c}$ \\
\hline & 800 & $80,2 b$ & $73,4 b$ & $77,0 \mathrm{~b}$ & $75,5 b$ & $78,8 \mathrm{~b}$ & $67,5 b$ \\
\hline & 1000 & $89,0 \mathrm{a}$ & $83,1 \mathrm{a}$ & $87,9 \mathrm{a}$ & $86,4 a$ & $89,5 a$ & $84,5 a$ \\
\hline \multirow[t]{5}{*}{$\mathrm{CaSO}_{4}, 2 \mathrm{H}_{2} \mathrm{O}$} & 200 & $15,9 \mathrm{e}$ & $8,0 \mathrm{e}$ & $9,5 \mathrm{e}$ & $6,7 \mathrm{e}$ & $6,8 \mathrm{e}$ & $7,0 \mathrm{e}$ \\
\hline & 400 & $26,8 \mathrm{~d}$ & $17,1 d$ & $18,0 \mathrm{~d}$ & $19,2 d$ & $18,7 d$ & $14,8 \mathrm{~d}$ \\
\hline & 600 & $33,6 \mathrm{c}$ & $23,7 \mathrm{c}$ & $26,8 \mathrm{c}$ & $25,9 c$ & $30,9 c$ & $23,6 c$ \\
\hline & 800 & $44,5 b$ & $30,3 b$ & $34,4 b$ & $34,7 b$ & $38,7 b$ & $30,4 b$ \\
\hline & 1000 & $50,9 a$ & $37,6 a$ & $32,6 a$ & $36,4 a$ & $40,4 a$ & $39,5 a$ \\
\hline \multirow[t]{5}{*}{$\mathrm{Ca}(\mathrm{ClO})_{2}$} & 200 & $6,6 \mathrm{~d}$ & $4,5 \mathrm{dd}$ & $3,6 \mathrm{e}$ & $3,0 \mathrm{e}$ & $4,2 c$ & $3,7 d$ \\
\hline & 400 & $7,8 \mathrm{~d}$ & $3,3 d$ & $4,7 \mathrm{de}$ & $3,5 d$ & $4,8 \mathrm{~d}$ & $6,8 \mathrm{c}$ \\
\hline & 600 & $10,0 \mathrm{c}$ & $5,6 \mathrm{c}$ & $7,4 \mathrm{~cd}$ & $5,8 \mathrm{c}$ & $6,7 \mathrm{c}$ & $8,3 b$ \\
\hline & 800 & $13,6 b$ & $7,6 \mathrm{~b}$ & $7,0 \mathrm{~b}$ & $9,5 b$ & $6,4 b$ & $8,2 b$ \\
\hline & 1000 & $16,3 a$ & $12,4 a$ & $10,5 a$ & $14,6 \mathrm{a}$ & $9,3 a$ & $13,4 a$ \\
\hline
\end{tabular}

Pour chaque isolat et chaque sel, deux résultats lus sur une même colonne diffèrent significativement au seuil de 5\% s'ils ne sont affectés d'aucune lettre en commun.

A. alternata: Alternaria alternata, A. niger: Aspergillus niger, F. oxysporum: Fusarium oxysporum, $\boldsymbol{P}$. digitatum: Penicillium digitatum, $\boldsymbol{R}$. stolonifer: Rhizopus stolonifer, $\boldsymbol{P}$.

italicum: Penicillium italicum 
Bulletin de la Société Royale des Sciences de Liège, Vol. 85, 2016, p. 263 - 275

Tableau $6: \mathrm{CI}_{50}$ et $\mathrm{CI}_{90}(\mathrm{ppm})$ de la germination des champignons testés en présence de composés calciques inorganiques.

\begin{tabular}{|c|c|c|c|c|c|c|c|c|c|c|c|c|}
\hline \multirow{3}{*}{$\begin{array}{l}\text { Sels de } \\
\text { calcium }\end{array}$} & \multicolumn{12}{|c|}{ Champignons } \\
\hline & \multicolumn{2}{|c|}{$\mathbf{A a}$} & \multicolumn{2}{|c|}{ An } & \multicolumn{2}{|c|}{ Pd } & \multicolumn{2}{|c|}{$\overline{\mathbf{P i}}$} & \multicolumn{2}{|c|}{ Fo } & \multicolumn{2}{|c|}{ Rs } \\
\hline & $\mathrm{CI}_{50}$ & $\mathbf{C I}_{90}$ & $\mathrm{CI}_{50}$ & $\mathrm{CI}_{90}$ & $\mathrm{CI}_{50}$ & $\mathrm{CI}_{90}$ & $\mathrm{CI}_{50}$ & $\mathrm{CI}_{90}$ & $\mathrm{CI}_{50}$ & $\mathbf{C I}_{90}$ & $\mathrm{CI}_{50}$ & $\mathbf{C I}_{90}$ \\
\hline Hydroxyde & 344 & $>1000$ & 282 & $>1000$ & 268 & $>1000$ & 294 & 912 & 284 & 759 & 286 & $>1000$ \\
\hline Sulfate & $>1000$ & $>1000$ & $>1000$ & $>1000$ & $>1000$ & $>1000$ & $>1000$ & $>1000$ & $>1000$ & $>1000$ & $>1000$ & $>1000$ \\
\hline Silicate & 312 & $>1000$ & 284 & $>1000$ & 331 & $>1000$ & 259 & $>1000$ & 230 & 925 & 290 & $>1000$ \\
\hline Oxyde & 298 & $>1000$ & 275 & $>1000$ & 274 & $>1000$ & 272 & $>1000$ & 323 & $>1000$ & 447 & $>1000$ \\
\hline Hypochlorite & $>1000$ & $>1000$ & $>1000$ & $>1000$ & $>1000$ & $>1000$ & $>1000$ & $>1000$ & $>1000$ & $>1000$ & $>1000$ & $>1000$ \\
\hline
\end{tabular}

Aa : Alternaria alternata, An :Aspergillus niger, Fo :Fusarium oxysporum, Pd:Penicillium digitatum, Rs:Rhizopus stolonifer, Pi :Penicillium italicum

\section{DISCUSSION ET CONCLUSION}

Le silicate, l'hydroxyde et l'oxyde de calcium s'avèrent très efficaces sur les trois stades de cycle de vie des isolats de Fusarium oxysporum, Alternaria alternata et de Trichothecium roseum. Selmaoui (1999) a rapporté que ces composés calciques inorganiques sont très efficaces dans l'inhibition de la croissance mycélienne, la production des conidies et la germination d'Alternaria alternata, Penicillium spp., Trichoderma spp., Trichothecium roseum et Fusarium avenaceum responsables des pourritures des pommes en conservation. Allantophomopsis cytisporea, A. lycopodina, Coleophoma empetri, Fusicoccum putrefaciens et Physalospora vaccinii, champignons responsables des pourritures des canneberges, sont très sensibles à l'action du silicate, l'hydroxyde et l'oxyde de calcium (Blodgett, 2002). Ces derniers ont pu inhiber in vitro de manière importante Monilia fructicola, responsable de la moniliose des pêches (Biggs et al., 1997). Ils sont utiles dans l'inhibition de la pourriture racinaire noire des carottes causée par Chalara elegans (Punja et Gaye, 1993). En présence du sulfate de calcium, la croissance mycélienne, la production des conidies et la germination des champignons testés sont sensiblement affectées. Des résultats similaires ont été signalés par Selmaoui (1999). Ce dernier a montré que ce composé calcique inorganique présente une efficacité moyenne ou faible vis-à-vis des trois stades de cycle de vie des champignons testés. L'hypochlorite de calcium n'a montré aucune action contre tous les espèces fongiques testées. Ces résultats rejoignent ceux de Biggs et al. (1994) qui ont signalé que l'hypochlorite de calcium est inefficace contre la pourriture de la pêche causée par Leucostoma persoonii. D'un autre côté, Martin-Diana et al.(2007) ont rapporté que le traitement par le calcium, en trempant les fruits dans des solutions de calcium, peut améliorer leur qualité nutritionnelle durant la conservation et accroître la durée de leur vie en maintenant leur fermeté. Il permet aussi de réduire le taux de la respiration et de la production d'éthylène, de retarder la maturation des fruits, de réduire la carie et le brunissement et d'atténuer les dégâts dus au froid. De même, Rao et al. (2011) ont signalé que le traitement du poivron par le $\mathrm{CaCl}_{2}$ permet de retarder la maturation, de prolonger la durée de vie et d'améliorer la qualité de leur 
conservation, tout en conservant la qualité nutritionnelle. Les résultats satisfaisants de cette étude permettront de suggérer l'utilisation des composés calciques inorganiques qui présentent un effet inhibiteur contre les pathogènes responsables des pourritures des agrumes en post-récolte, avant l'entreposage, en raison de son efficacité contre les maladies d'origine fongiques.

\section{Références}

Aharoni Y., Fallik E., Copel A., Gil M., Grinberg S. et Klein J.D. 1997. Sodium bicarbonate reduces postharvest decay development on melons. Postharv. Biol. Technol., 10(3), 201-206.

Anonyme, 2003. Notes de l'Office national de mise en valeur agricole du Souss-Massa. Service de production agricole, Maroc, $15 \mathrm{p}$.

Arslan U., Ilhan K. et Karabulut O.A. 2009. Evaluation of antifungal activity of food additives against soil borne phytopathogenic fungi. World J. Microbiol. Biotechnol., 25: 537543.

Arslan U., Ilhan K. et Karabulut O.A. 2006. Evaluation of food additives and low-toxicity compounds for the control of bean rust and wheat leaf rust. J. Phytopathol., 154:534-541.

Askame L., Talibi I., Boubaker H., Serghini O. A., Boudyach E. H. et Ait Ben Aoumar A. 2011.-Effects of Organic Acids and Salts on the Development of Penicillium italicum: The Causal Agent of Citrus Blue Mold, Plant Pathol. J.

Bancroft M.C., P.D. Gardner, J.W. Eckert \& J.L. Baritelle, 1984. Comparison of decay control strategies in California lemon packing houses. Plant Diseases, 68, 24-28.

Bi Y., Tian S.P., Guo Y.R., Ge Y.H. et Qin G.Z. 2006. Sodium silicate reduces postharvest decay on Hami melons: Induced resistance and fungistatic effects. Plant Dis., 90(3), 279-283.

Biggs A.R., El-Kholi M.M. et El-Neshawy S.M. 1994. Effect of calcium salts on growth, pectic enzyme activity, and colonization of Peach twigs by Leucostoma persoonii. Plant Dis., 78(9), 886-890.

Biggs A.R., El-Kholi M.M., El-Neshawy S. et Nickerson R. 1997. Effects of calcium salts on growth, polygalacturonase activity and infection of peach fruit by Monilia fructicola. Plant Dis., 81(4), 399-403.

Blodgett A.B., Caldwell R.W. et McManus P.S. 2002. Effects of calcium salts on the cranberry fruit rot disease complex. Plant Dis., 86(6), 747-752.

Bokshi A.I. 2008. Postharvest disease control of melons using systemic resistance and other. PhD. Univ. Sydney, Fac. Agric., Food Nat. Resources, 255p. 
Bus V.G., A.J. Bongers \& LA. Risse, 1997. Occurrence of Penicillium digitatum and Penicillium italicum resistant to benomyl, thiabendazole, and imazalil on citrus fruit from different geographic origin. Plant Diseases, 75, 1098-1100.

Chalutz E. \& C.L. Wilson, 1990. Postharvest biocontrol of Green and blue and sour rot of citrus fruit by Debaryomyces hansenii. Plant Diseases, 74, 134-137.

Droby S. E. Chalutz, C.L. Wilson \& M.E. Wisniewski, 1989. Characterization of the biocontrol activity of Debaryomyces hansenii in the control of Penicillium

digitatum on grape-fruit. Can. J. Microbiol., 35, 794-800.

Eckert J.W., 1990. Impact of fungicide resistance on citrus fruit decay control. In: Managing resistance to agrochemicals. M.B. Green, H.M. LeBaron \& W.K.

Moberg (eds.), American Chemical Society, Washington DC, 286-302.

Eckert J.W. \& I.L. Eaks, 1989. Postharvest disorders and diseases of citrus fruits. In: The Citrus Industry, 4. E.C. Calavan \& G.E. Carman (eds.), University of California Press, Berkeley, 179-269.

Eckert J.W., J.R Sievert. \& M. Ratnayake, 1994. Reduction of imazalil effectiveness against citrus green mold in California packinghouses by resistant biotypes of Penicillium digitatum. Plant Diseases, 78, 971-974.

El-Goorani M.A., H.M. El-Kascheir, M.T. Kabeel \& A.A. Shoeib, 1984. Resistance to benzimidazole of Penicillium italicum and Penicillium digitatum isolated from packinghouses and orchards in Egypt. Plant

Diseases, 68, 100-102.

Elkhamass M., B. Oulahcen, A. Lekchiri, A. Sebbata \& Y. Charhabaili, 1994. Stratégie de lutte contre les maladies de post-récolte des fruits d'agrumes, In:

Postharvest pathology and technology for horticultural commodities: recent advances. A. Ait Oubahou \& M. El-Otmani (eds.), Institut agronomique et vétérinaire Hassan II, Agadir, Maroc, 388-398.

Khamis Y., Ligorio A., Sanzani S. M., Nigroal F., _Ippolito A. 2012. Control of storage diseases of Citrus by pre- and postharvest applications of salts, Postharvest Biol. Technol. 72: 57-63.

Martin-Diana A.B., Rico D., Frias J.M., Barat J.M., Henehan G.T.M. et Barry-Ryan C. 2007. Calcium for extending the shelf life of fresh whole and minimally processed fruits and vegetables: a review. Trend Food Sci. Technol., 18, 210-218. 
Miller W.R., D. Chun, L.A. Risse, T.T. Hatton \& T. Hinsch, 1988. Influence of selected fungicide treatments to control the development of decay in waxed or film wrapped Florida grapefruit. Proceedings of the sixth International Citrus Congress, R. Goren \& K. Mendel (eds.), Balaban Publishers, Philadelphia, 1471-1477.

Nigro F., Schena, Ligorio, I. Pentimone L., Ippolito A. et Salerno M.G. 2006. Control of table grape storage rots by pre-harvest applications of salts. Post harvest Biol. Technol., 42: 142149.

Punja Z.K. et Gaye M.M. 1993. Influence of postharvest handling practices and dip treatments on development of black root rot on fresh market carrots. Plant Dis., 77(10), 989995.

Rao T.V.R., Gol N.B. et Shah K.K. 2011. Effect of postharvest treatments and storage temperatures on the quality and shelf life of sweet pepper (Capsicum annum L.). Sci. Hortic., 132, 18-26.

Selmaoui K. 1999. Étude d'un complexe fongique responsable de la pourriture des pommes en conservation. Application de quelques moyens de lutte chimique. Thèse Doctorat, Univ. Ibn Tofaïl, Fac. Sci. Kénitra, Maroc, 175 p.

Smilanick J.L., Margosan D.A., Mlikota F., Usall J. et Michael I.F. 1999. Control of citrus green mold by carbonate and bicarbonate salts and the influence of commercial postharvest practices on their efficacy. Plant Dis., 83(2), 139-145. http://www.mtco. org/doc/Mexico1.psd.pdf

Troncoso-Rojas R., Corral-Acosta Y., Sánchez-Estrada A., Garcia-Estrada R., AguilarValenzuela A., Ojeda-Contreras J. et Tiznado-Hernández M.E. 2009. Postharvest treatment of isothiocyanates to control Alternaria rot in netted melon. Phytoparasitica, 37(5), 445-451.

Zemmouri F., El Mhadri M., Selmaoui K., Benkirane R., Ouazzani Touhami A., Badoc A. et Douira A. 2011. Effet de sels de calcium sur les pourritures du melon en post-récolte. Bull. Soc. Pharm. Bordeaux, 150(1-4), 107-122 